\title{
Tooth Mortality: An Analysis of Reasons underlying the Extraction of Permanent Teeth
}

\author{
${ }^{1}$ Varsha Sunil Manekar, ${ }^{2}$ Prajwalit Kende, ${ }^{3}$ Seema Kulkarni
}

\begin{abstract}
The patients loose tooth/teeth for various reasons at different year of age. The most common reason are caries and periodontal disease. There is debate on the main cause out of these two. This may vary depending on other variables like age, food habits, general condition. The other causes of tooth extraction are failure of endodontics, fracture, infection. Some disease free teeth need to be sacrificed for orthodontic or prosthetic treatment plan. There is need of evaluation of reasons for to oth mortality. This will be the indicator of status of oral health awareness among population and success of oral healthcare delivered. In a prospective survey at our department collected data of 1186 subjects undergoing extractions of tooth/teeth. This data were evaluated for age and gender distribution, prevalence of reason for extraction and that of each tooth type in both arches.
\end{abstract}

Aim: The aim of survey is to study of prevalence and reasons of extraction of teeth according to age groups. In this prospective survey, the data were collected from the outpatient registration desk of extraction room of department of oral and maxillofacial surgery of G overnment Dental C ollege and Hospital, Mumbai, India. The assessment of the data was done by a single senior faculty. The data were analyzed for age, gender, reason for extraction and tooth/teeth extracted. Total 1,568 extractions were performed on 1,186 subjects.

Conclusion: The dental caries and its sequel is the principal reason for extraction of teeth followed by periodontal disease. The first molar of both arches is the tooth extracted most frequently for caries and its sequel in young age group of 15 to 25 years.

Keywords: Extraction, Caries, Causes of extraction, First molar, Orthodontic reason, Periodontal disease, Reasons of extraction, Tooth mortality, Young patient.

How to cite this article: Manekar VS, Kende $P$, Kulkarni $S$. Tooth Mortality: An Analysis of Reasons underlying the Extraction of Permanent Teeth. World J Dent 2015;6(2):93-96.

\section{Source of support: Nil}

Conflict of interest: None

\footnotetext{
${ }^{1}$ Associate Professor, ${ }^{2}$ Assistant P rofessor, ${ }^{3}$ Dental Surgeon

${ }^{1}$ Department of Oral and Maxillofacial Surgery, Government Dental College and Hospital, Nagpur, Maharashtra, India

2,3Department of Oral and Maxillofacial Surgery, Government Dental College and Hospital, Mumbai, Maharashtra, India
}

Corresponding Author: Varsha Sunil Manekar, Associate Professor, Department of Oral and Maxillofacial Surgery, Government Dental College and Hospital, Nagpur, Maharashtra, India Phone: 9823077626, e-mail: varsha manekar@yahoo.co.in

\section{INTRODUCTION}

Teeth are irreplaceable gifts for each individual. But, the dentistry was considered equal to extraction of teeth. Over the years as the dentistry evolved the concept of conservation of tooth became widely acceptable. Tooth retention is improving because of better prevention and control of the oral diseases, more positive attitudes toward tooth retention, and more conservative dental treatment philosophies. Does that mean the tooth extraction procedure is out of practice? Of course, 'No'. In spite of an increasing awareness of the value of preventive measures to maintain oral health, the effects of caries and periodontal disease are not always dealt with in a timely manner. The presence of pain to alert the individual of a pending dental problem is, in addition, rather the exception than the rule. ${ }^{1}$ It is also suggested that the analysis of tooth mortality statistics is important in evaluating dental care, implying that tooth mortality in dentistry is analogous with mortality statistics in the medical field. ${ }^{2}$

The patients loose tooth/teeth for various reasons at different year of age. The most common reason are caries and periodontal disease. There is debate on the main cause out of these two. This may vary depending on other variables like age, food habits, general condition. The other causes of tooth extraction are failure of endodontics, fracture, infection. Some disease free teeth need to be sacrificed for orthodontic or prosthetic treatment plan. There is need of evaluation of reasons for tooth mortality. This will be the indicator of status of oral health awareness among population and success of oral health care delivered. The data of 1,186 subjects undergoing extractions of tooth/teeth were collected. This data were evaluated for age and gender distribution, prevalence of reason for extraction and that of each tooth type in both arches.

\section{AIM}

The aim of survey is to study of prevalence and reasons of extraction of teeth according to age groups.

\section{OBJ ECTIVES}

- Distribution of extraction cases in decided age groups (A-G). 
- Cases distribution according to gender in the age groups.

- Prevalence of reasons of extraction.

- Prevalence of extraction of each tooth type in both arches.

\section{METHODS}

Sample collection: The data were collected from the extraction room of department of oral and maxillofacial surgery of Government Dental College and Hospital, Mumbai, India. Duration of survey was 1 month, from 17/7/2012 to $17 / 8 / 2012$. Information was collected at the outpatient registration desk: age, gender, reason for extraction and tooth/teeth extracted were noted. The patients were put in age groups $A$ to $G$ as shown in Table 1.

The reasons for teeth mortality were adapted based on Cahen et $\mathrm{al}^{3}$ with little modification. The assessment of the data was done by a single senior faculty. The data of reasons for tooth extraction were put in following categories:

- Caries-carious tooth as well their sequels including all endodontic reasons

- Periodontal diseases-tooth extraction for all periodontal pathologies

- Orthodontic reason-tooth indicated for extraction as part of orthododntic treatment planning

- Trauma - tooth extracted for external trauma

- Over retained deciduous teeth

Impacted teeth and third molar extraction were not included in sample collection. The tooth type and arch was also noted.
The data, thus, collected were put in Tables 1 to 4 for analysis. Table 1 shows the distribution of subjects according to age groups $A$ to $G$ and according to gender in each group. Table 2 shows the prevalence of reasons of tooth extraction in groups A to G. The prevalence of maxillary tooth in age groups A to $\mathrm{G}$ is in Table 3. Table 4 shows the prevalence of mandibular tooth in age groups A to $\mathrm{G}$.

\section{Observation}

The assessment of data was done and analyzed as follows:

- Out of 1,186 subjects, the distribution in age groups and gender is shown in Table 1. Maximum teeth are lost by age group A that is in 15 to 25 years $20.74 \%$, followed by group B (17.11\%) and C (18.04\%). In group $\mathrm{D}$ and $\mathrm{E}$, almost equal number of extractions were performed that is 16.35 and $16.10 \%$ respectively. The group $\mathrm{F}$ and $\mathrm{G}$ underwent least extractions 8.51 and $2.95 \%$ respectively.

- From Table 1, groups A and C have almost equal gender distribution. In group B, female percentage was more than male patients, whereas male percentage of extraction was greater in groups D, E, F and G age groups. Graph 1 shows the findings clearly.

- Total 1,568 extractions were performed on 1,186 subjects. Table 2 shows the prevalence of reasons for extractions. The caries was the most common reason with $55.67 \%$ teeth extracted for this cause. Second most common cause being periodontal reasons with $23.59 \%$. The $18.17 \%$ cases of retained tooth removed.

Table 1: Subjects with age groups and gender ratio

\begin{tabular}{llllllll}
\hline $\begin{array}{l}\text { Age } \\
\text { groups }\end{array}$ & $\begin{array}{l}\text { Age range } \\
\text { (years) }\end{array}$ & $\begin{array}{l}\text { Number of } \\
\text { extractions }\end{array}$ & Percentage & Male & Percentage & Female & Percentage \\
\hline A & $15-25$ & 248 & 20.74 & 121 & 48.79 & 127 & 51.20 \\
B & $26-35$ & 203 & 17.11 & 80 & 39.40 & 123 & 60.59 \\
C & $36-45$ & 214 & 18.04 & 104 & 48.59 & 110 & 51.40 \\
D & $46-55$ & 194 & 16.35 & 117 & 60.30 & 77 & 39.69 \\
E & $56-65$ & 191 & 16.10 & 131 & 68.58 & 60 & 31.41 \\
F & $66-75$ & 101 & 8.51 & 77 & 76.23 & 26 & 25.74 \\
G & $76-85$ & 35 & 2.95 & 24 & 68.57 & 11 & 31.42 \\
\hline
\end{tabular}

Table 2: Reasons for tooth extraction

\begin{tabular}{llllllllll}
\hline Reasons & A & B & C & D & E & F & G & Total & Percentage \\
\hline Caries & 230 & 190 & 182 & 131 & 97 & 35 & 8 & 873 & 55.67 \\
Periodontal & 9 & 14 & 51 & 63 & 126 & 76 & 31 & 370 & 23.59 \\
$\begin{array}{l}\text { Ortho } \\
\text { Trauma }\end{array}$ & 16 & & & & & & & 16 & 1.02 \\
$\begin{array}{l}\text { Retained } \\
\text { deciduous }\end{array}$ & 10 & & 3 & 3 & 3 & & 11 & 0.70 \\
\hline
\end{tabular}


Tooth Mortality: An Analysis of Reasons underlying the Extraction of Permanent Teeth

Table 3: Prevalence of maxillary tooth in age groups $A$ to $G$

\begin{tabular}{llllllllll}
\hline Teeth no. & A & B & C & D & E & F & G & Total & Percentage \\
\hline Central incisors & 8 & 2 & 3 & 15 & 15 & 10 & 4 & 57 & 3.63 \\
Lateral incisors & 4 & 11 & 14 & 13 & 23 & 18 & 6 & 88 & 5.61 \\
Canine & - & 5 & 15 & 9 & 23 & 17 & 6 & 75 & 4.78 \\
First PM & 17 & 17 & 36 & 23 & 21 & 8 & 6 & 128 & 8.16 \\
Second PM & 14 & 21 & 24 & 28 & 19 & 11 & 1 & 118 & 7.5 \\
First M & 62 & 33 & 26 & 32 & 25 & 18 & 6 & 202 & 12.88 \\
Second M & 11 & 24 & 19 & 27 & 26 & 8 & 4 & 119 & 7.5 \\
\hline
\end{tabular}

Table 4: Prevalence of mandibular tooth in age groups $A$ to $G$

\begin{tabular}{llllllllll}
\hline Teeth no. & A & B & C & D & E & F & G & Total & Percentage \\
\hline Central incisor & 0 & 2 & 10 & 10 & 15 & 7 & 1 & 45 & 2.86 \\
Lateral incisor & 0 & 3 & 10 & 13 & 20 & 7 & 7 & 60 & 1.31 \\
Canine & 2 & 3 & 12 & 11 & 19 & 9 & 3 & 59 & 3.76 \\
First PM & 4 & 8 & 14 & 21 & 23 & 5 & 2 & 77 & 4.91 \\
Second PM & 17 & 17 & 17 & 19 & 30 & 9 & 3 & 112 & 7.14 \\
First M & 120 & 57 & 48 & 30 & 35 & 5 & 4 & 299 & 19.06 \\
Second M & 27 & 33 & 16 & 17 & 21 & 12 & 3 & 129 & 8.22 \\
\hline
\end{tabular}

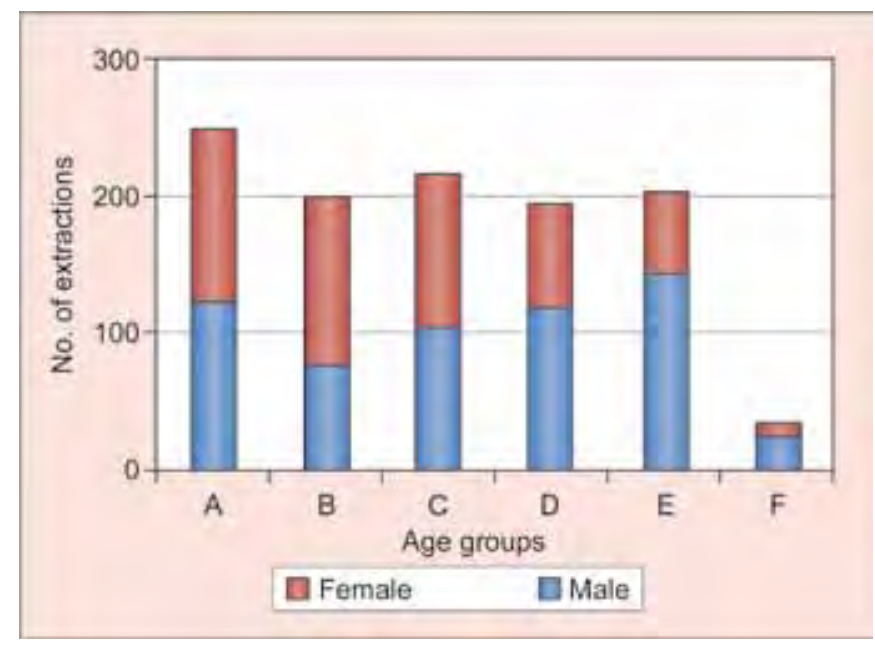

Graph 1: Age and gender

As seen from Graph 2, group A has maximum number of extractions for caries reason and group $\mathrm{E}$ has maximum number of extractions for periodontal reasons.

Caries is the reason most prevalent for group A, followed by B and C. Thus, dental caries is the most common cause of tooth loss in the subjects under age 45 and the periodontal disease was the major cause for tooth loss in patients over 45 years old. Ortho extractions are done in A group.

- Among the maxillary teeth, individual tooth extraction analysis as seen in Table 3, the first molar was extracted with highest percentage of $12.88 \%$. The maximum first molars were extracted in age group A. The canine is the tooth extracted in minimum number of patients.

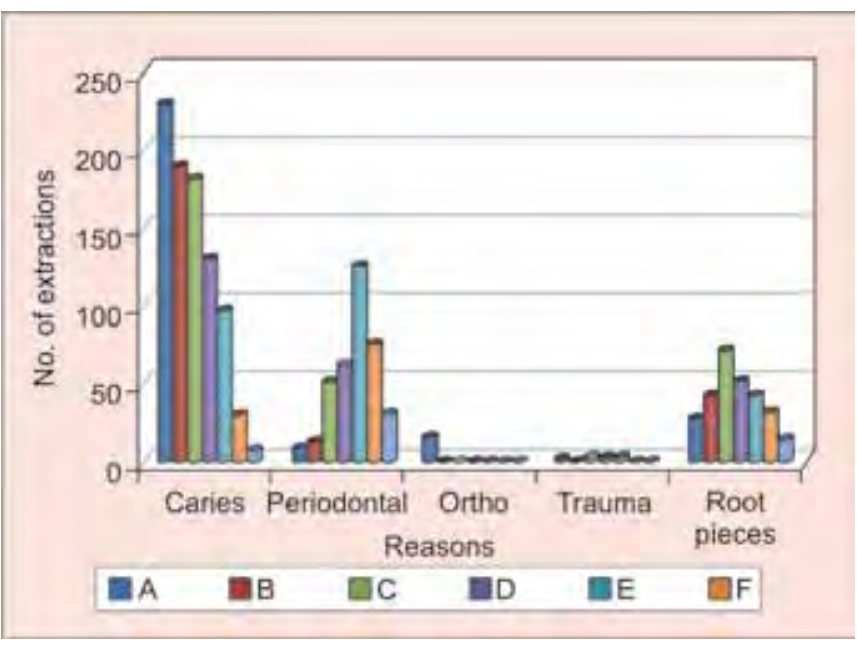

Graph 2: Reasons of extraction

- Similarly, Table 4 shows comparison of individual extractions of mandibular teeth. The first molar was the most commonly extracted tooth with $19.06 \%$, followed by second molar, second premolar, first premolar and canine. The central and lateral incisors are with least percentage.

- Highest number of teeth extracted is the mandibular first molar tooth and that is in age group A.

- There is almost equal distribution of extraction number in both arches: maxillary-50.19\% and mandibular-49.80\%.

\section{RESULTS}

Maximum teeth are extracted in age group A that is in 15 to 25 years: $20.74 \%$. The caries was the most common 
reason with $55.67 \%$ teeth extracted for this cause. The first molars of both arches were with highest percentage of extraction and the age group A was with maximum number of first molar extraction.

\section{DISCUSSION}

Understanding the reasons for tooth loss is important in the formulation of strategies and the planning of dental health services, as well in providing information about the prevalence of dental diseases and the availability of dental care. World Health Organization states good oral health as an indicator of good health and one of the global goals to be achieved by the year 2000 is $85 \%$ of the population should have all teeth by the age of 18 years. ${ }^{4}$

The literature indicates dental caries as the most common reason for loss of tooth. ${ }^{6}$ In our prospective survey of patients undergoing extraction nearly half of the teeth (55.67\%) were extracted due to dental caries and its sequel, whereas more than one third teeth extracted due to periodontal disease $(23.59 \%)$. This finding is consistent with findings from other studies where dental caries is the main reason for tooth loss over all followed by periodontal disease. Studies investigating the reasons for tooth extraction have been carried out in many countries, mostly in developed industrial Europe. The results of these studies showed that dental caries and periodontal disease were the most common causes of tooth loss. Dental caries was the most important cause of tooth loss in the population under the age 40 years and the periodontal disease was the major cause for tooth loss in patients over 40 years old. ${ }^{5}$ Distribution by age group and dental diseases is highly significant. The patients in age group A (15-25 years) had maximum teeth extracted for caries followed by other younger age groups B and C. Age group E (56-65 years) have maximum teeth extracted due to periodontal diseases, followed by age group D. This is consistent to a study conducted in Scotland where the proportion of extractions attributed to periodontal disease increased from age 31 to 60 years. ${ }^{7}$
This study was carried out in the Government Dental College and Hospital, Mumbai, India. It indicates the prevalence of extraction as treatment with distribution of age, reasons, and disease among patients reporting to our department. The present study shows that dental caries is the leading cause of extraction in younger groups with periodontal disease predominating in older groups. It shows that the mandibular first molar is the most commonly extracted tooth in the younger age for caries. First molar is the first permanent teeth to erupt in the oral cavity and is at high risk of dental caries in the absence of appropriate preventive measures.

\section{CONCLUSION}

The dental caries and its sequel is the principal reason for extraction of teeth followed by periodontal disease. Among the distribution of age, reasons, and disease first molar of both arches is the tooth extracted most frequently for caries and its sequel in young age group of 15 to 25 years.

\section{REFERENCES}

1. Taintor JF, Langeland K, Valle GF, Krasny RM. Pain: a poor parameter of evaluation in dentistry. Oral Surg Oral Med Oral Path 1981;3:299-303.

2. Richards W, Ameen J, Coll AM, Higgs G. Reasons for tooth extraction in four general dental practices in South Wales. Br Dent J 2005;198(5):275-278.

3. Cahen PM, Frank RM, Turlot JC. A survey of the reasons for dental extractions in France. J Dent Res 1985;64(8):1087-1093.

4. The World Health Report 2000: Continuous improvement of oral health in the 21st century-the approach of the WHO Global Oral Health Program. Community Dent Oral Epidemiol 2003;(Suppl 1):3-21.

5. Wahab FK. Survey of reasons for extraction of permanent teeth in Jord Saudi Dent J 2001 Sep-Dec;13(3):148-154.

6. Shetye O, Shetye NK, Louis A, Roy I. Immediate implantation in an extraction socket: a review. Int J Contemp Dent Med Rev 2015;2015: Article ID: 160115. DOI: 10.15713/ins.ijcdmr.39

7. Iwasaki, Masanori, Nakamura K, Yoshihara A, Miyazaki H. Change in bone mineral density and tooth loss in Japanese community-dwelling postmenopausal women: a 5-year cohort study. J Bone and Mineral Metabolism 30, no. 4 2012:447-453. 